\title{
Teores foliares de macro e micronutrientes em milho tolerante ao glyphosate submetido à herbicidas
}

\author{
Foliar levels of macro and micronutrients in glyphosate-tolerant \\ corn submitted to herbicides
}

\author{
Núbia Maria Correia ${ }^{1 *}$; Edson Aparecido dos Santos ${ }^{2}$
}

\begin{abstract}
Resumo
Uma série de fatores relacionados ao desenvolvimento do milho tolerante ao glyphosate devem ser abordados e melhor estudados. Com o objetivo de avaliar o efeito de tratamentos herbicidas nos teores foliares de $\mathrm{N}, \mathrm{P}, \mathrm{K}, \mathrm{Ca}, \mathrm{Mg}, \mathrm{S}, \mathrm{B}, \mathrm{Cu}, \mathrm{Fe}, \mathrm{Mn}$ e zinco $\mathrm{Zn}$ e na produção de grãos de milho cultivar híbrido DKB390 RR tolerante ao herbicida glyphosate, foi desenvolvido experimento em condições de campo, no ano agrícola 2010/2011. O delineamento experimental foi o de blocos ao acaso, com 12 tratamentos e quatro repetições. Os herbicidas glyphosate (720, 1200 e sequencial de 960 mais 720 $\mathrm{g} \mathrm{ha}^{-1}$ de equivalente ácido), atrazine (2500 $\left.\mathrm{g} \mathrm{ha}^{-1}\right)$, nicosulfuron $\left(60 \mathrm{~g} \mathrm{ha}^{-1}\right)$, mesotrione $\left(192 \mathrm{~g} \mathrm{ha}^{-1}\right)$, tembotrione $\left(100,8 \mathrm{~g} \mathrm{ha}^{-1}\right)$, atrazine mais glyphosate $\left(1000+960 \mathrm{~g} \mathrm{ha}^{-1}\right)$, atrazine mais nicosulfuron $\left(1000+20 \mathrm{~g} \mathrm{ha}^{-1}\right)$, atrazine mais mesotrione $\left(1000+144 \mathrm{~g} \mathrm{ha}^{-1}\right)$ e atrazine mais tembotrione $(1000+$ $75,6 \mathrm{~g} \mathrm{ha}^{-1}$ ) e uma testemunha sem aplicação foram estudados. Os herbicidas glyphosate e mesotrione isolados não causaram nenhuma injúria visível às plantas. Os demais herbicidas ocasionaram sintomas visuais de fitointoxicação muito leve $(<5 \%)$. Os tratamentos não afetaram os teores foliares de $\mathrm{N}, \mathrm{P}$, $\mathrm{K}, \mathrm{Ca}, \mathrm{Mg}, \mathrm{S}, \mathrm{B}, \mathrm{Mn}$ e $\mathrm{Zn}$. Mas, as plantas tratadas com atrazine mais nicosulfuron tiveram maior teor foliar de Fe, e aquelas pulverizadas com glyphosate (nas três dosagens) e atrazine, maior acúmulo de $\mathrm{Cu}$ nas folhas. A aplicação isolada de glyphosate $\left(1200 \mathrm{~g} \mathrm{ha}^{-1}\right)$, nicosulfuron, tembotrione e da mistura atrazine mais nicosulfuron ocasionaram redução na produção de grãos.
\end{abstract}

Palavras-chave: Milho Roundup Ready, milho resistente ao glyphosate, nutrição mineral

\begin{abstract}
A serial of factors related to development of glyphosate-tolerant corn should be approached and best studied. This work was developed to evaluate foliar levels of N, P, K, Ca, Mg, S, B, Cu, Fe, Mn and Zn, besides the grain yield of glyphosate-tolerant corn (DKB390 RR hybrid). An experiment was carried out under field conditions, during the crop year of 2010/2011. A randomized complete block design with four repetitions was used to distribute the treatments in the field. Twelve herbicide treatments were studied: glyphosate $\left(720,1200\right.$ and sequential application of 960 plus $720 \mathrm{~g} \mathrm{ha}^{-1}$ of the acid glyphosate equivalent), atrazine (2500 $\left.\mathrm{g} \mathrm{ha}^{-1}\right)$, nicosulfuron $\left(60 \mathrm{~g} \mathrm{ha}^{-1}\right)$, mesotrione $\left(192 \mathrm{~g} \mathrm{ha}^{-1}\right)$, tembotrione $(100,8$ $\left.\mathrm{g} \mathrm{ha}^{-1}\right)$, atrazine plus glyphosate $\left(1000+960 \mathrm{~g} \mathrm{ha}^{-1}\right)$, atrazine plus nicosulfuron $\left(1000+20 \mathrm{~g} \mathrm{ha}^{-1}\right)$, atrazine plus mesotrione $\left(1000+144 \mathrm{~g} \mathrm{ha}^{-1}\right)$ and atrazine plus tembotrione $\left(1000+75,6 \mathrm{~g} \mathrm{ha}^{-1}\right)$ and one control treatment without herbicide. The glyphosate and mesotrione alone did not cause no visible injury to corn. The other herbicides caused intoxication symptoms classified as light $(<5 \%)$. The treatments studied did not affect foliar levels of N, P, K, Ca, Mg, S, B, Mn and $\mathrm{Zn}$ in the corn plants. But, the plants treated with atrazine plus nicosulfuron had more leaf Fe content, and the plants sprayed with glyphosate
\end{abstract}

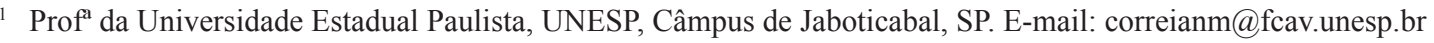

2 Pesquisador, Empresa Brasileira de Pesquisa Agropecuária, EMBRAPA Milho e Sorgo, Sete Lagoas, MG. E-mail: edsonapsant@ yahoo.com.br

Autor para correspondência 
(in the three doses) and atrazine, more accumulation of $\mathrm{Cu}$ in the leaf. The Grain yield of corn was reduced with application single of glyphosate $\left(1200 \mathrm{~g} \mathrm{ha}^{-1}\right)$, nicosulfuron, tembotrione and with the mixture atrazine plus nicosulfuron.

Key words: Roundup Ready corn, glyphosate-resistant corn, mineral nutrition

\section{Introdução}

No Brasil, há 135 herbicidas registrados para a cultura do milho, em que a maioria é seletiva às plantas (AGROFIT, 2011). Dentre os produtos pulverizados em pós-emergência, os ingredientes ativos atrazine, mesotrione, nicosulfuron e tembotrione são os mais utilizados. No entanto, com a liberação do cultivo do milho tolerante ao glyphosate no Brasil, em 2010, o uso de herbicidas nessa cultura sofrerá profunda modificação. Por outro lado, há uma série de fatores relacionados ao desenvolvimento do milho transgênico que devem ser abordados e melhor estudados.

$\mathrm{Na}$ cultura da soja transgênica tolerante ao glyphosate, cultivada no Brasil desde 2005, constatou-se que o glyphosate interferiu negativamente na eficiência nutricional das plantas e nos teores totais de $\mathrm{N}, \mathrm{Mn}, \mathrm{Cu}, \mathrm{Zn}$ e Fe (SERRA et al., 2011). O mesmo ocorreu em outro trabalho para os teores foliares de N, Ca, Fe e Cu (SANTOS et al., 2007). O glyphosate afeta a absorção e a translocação desses nutrientes, possivelmente, pela ligação e formação de complexos insolúveis com o herbicida, imobilizando os elementos (CAKMAK et al., 2009).

A aplicação de glyphosate nos estágios vegetativos e início do reprodutivo da soja, quando a assimilação de nitrato é maior e menos sensível ao herbicida, também podem explicar o efeito no acúmulo de N (BELLALOUI et al., 2008). Porém, para Zablotowicz e Reddy (2007), as injúrias visuais observadas nas plantas de soja pulverizadas com glyphosate são oriundas da formação de aminometilfosfonico (AMPA), a partir da degradação de glyphosate nas plantas. A dimensão dos danos dependerá da concentração de AMPA, que será em função da dose utilizada de glyphosate, do genótipo de soja e das condições ambientais (ZABLOTOWICZ; REDDY, 2007).
Em plantas de milho tolerante, o glyphosate inibiu a atividade da enzima nitrato redutase, porém, o teor foliar de $\mathrm{N}$ e a produção de grãos não foram afetados (REDDY; BELLALOUI; ZABLOTOWICZ, 2010). Nesse mesmo trabalho, a aplicação de subdose de glyphosate em milho convencional, não transgênico, ocasionou reduções substanciais na atividade da nitrato redutase (de 46 a 64\%), na produtividade (de 49 a 54\%) e no teor de $\mathrm{N}$ nas folhas (de 7 a $16 \%$ ).

Por outro lado, outros herbicidas também podem comprometer a absorção, a translocação e/ou o metabolismo de nutrientes pelas plantas tratadas. Contudo, embora os herbicidas atrazine, nicosulfuon, mesotrione e tembotrione, sejam utilizados com frequência no campo, há uma carência de informações sobre o efeito dos mesmos na nutrição mineral das plantas de milho.

Com base na hipótese de que os teores de macro e micronutrientes em milho transgênico tolerante ao glyphosate podem ser afetados pela exposição das plantas aos herbicidas glyphosate, atrazine, mesotrione e tembotrione, isolados e em mistura, com reflexo na produção de grãos, objetivou-se estudar o efeito de tratamentos de herbicidas nos teores foliares desses macro e micronutrientes e na produção de grãos de milho cultivar DKB390 RR tolerante ao herbicida glyphosate pulverizado com diferentes tratamentos de herbicidas.

\section{Material e Métodos}

O experimento foi desenvolvido em condições de campo, no ano agrícola 2010/2011. O solo da área experimental é de textura argilosa, cuja análise química indicou $\mathrm{pH}$ em $\mathrm{CaCl}_{2}$ de 5,4; CTC, soma de bases, $\mathrm{H}+\mathrm{Al}, \mathrm{Ca}, \mathrm{Mg}$ e $\mathrm{K}$ de 26, 25, 10 e 3,7 $\mathrm{mmol}_{\mathrm{c}} \mathrm{dm}^{-3}$, respectivamente; $18 \mathrm{~g} \mathrm{dm}^{-3}$ de matéria orgânica e $31 \mathrm{mg} \mathrm{dm}^{-3}$ de P. 
O cultivar híbrido DKB390 RR foi semeado sob sistema de preparo convencional de solo, com espaçamento de $0,9 \mathrm{~m}$ entre linhas e 7,0 sementes por metro linear, em 01/12/2010. No sulco de semeadura foram aplicados $345 \mathrm{~kg} \mathrm{ha}^{-1}$ do formulado 04-20-20.

Vinte e dois dias após a semeadura do milho, em 23/12/2010, as plantas receberam a adubação nitrogenada em cobertura, na quantidade de $83 \mathrm{~kg}$ ha ${ }^{-1}$ de nitrogênio na forma de uréia.

O delineamento experimento foi o de blocos ao acaso, com 12 tratamentos e quatro repetições. Os herbicidas glyphosate (720, 1200 e sequencial das doses 960 e $720 \mathrm{~g} \mathrm{ha}^{-1}$ de equivalente ácido), atrazine (2500 $\left.\mathrm{g} \mathrm{ha}^{-1}\right)$, nicosulfuron (60 $\left.\mathrm{g} \mathrm{ha}^{-1}\right)$, mesotrione (192 $\left.\mathrm{g} \mathrm{ha}^{-1}\right)$, tembotrione $\left(100,8 \mathrm{~g} \mathrm{ha}^{-1}\right)$, atrazine mais glyphosate $\left(1000+960 \mathrm{~g} \mathrm{ha}^{-1}\right)$, atrazine mais nicosulfuron $\left(1000+20 \mathrm{~g} \mathrm{ha}^{-1}\right)$, atrazine mais mesotrione $\left(1000+144 \mathrm{~g} \mathrm{ha}^{-1}\right)$ e atrazine mais tembotrione $\left(1000+75,6 \mathrm{~g} \mathrm{ha}^{-1}\right)$ e uma testemunha sem aplicação foram estudados.

As parcelas foram constituídas por quatro linhas com sete metros de comprimento cada, totalizando 25,2 $\mathrm{m}^{2}$ de área total, com 10,8 $\mathrm{m}^{2}$ de área útil (duas linhas centrais com seis metros de comprimento cada).

Os herbicidas foram aplicados em pósemergência das plantas, em 19/12/2010 entre 9:05 e 10:20 horas. Utilizou-se pulverizador costal, à pressão constante (mantida pelo $\mathrm{CO}_{2}$ comprimido) de $25 \mathrm{lbf} \mathrm{pol}^{-2}$, munido de barra com seis bicos de jato plano XR 11002 distanciados em 0,5 m, com consumo de calda equivalente a $200 \mathrm{~L} \mathrm{ha}^{-1}$. As condições edafoclimáticas eram: temperatura do ar de 25,8 a $27,8^{\circ} \mathrm{C}$, umidade relativa do ar de 87 a $80 \%$, nebulosidade de 60 a $40 \%$, sem vento e solo com boa umidade. As plantas de milho tinham de 4 a 5 folhas totalmente desenroladas e de 25 a 30 $\mathrm{cm}$ de altura (do dossel). No tratamento indicado, a aplicação sequencial de glyphosate foi realizada 10 dias após a primeira, em 29/12/2010, utilizandose a mesma tecnologia descrita anteriormente. No momento da aplicação foram registrados $27,4^{\circ} \mathrm{C}$ de temperatura do ar, $74 \%$ de umidade relativa, céu aberto, velocidade do vento de 0,8 a $1,0 \mathrm{~km} \mathrm{~h}^{-1}$ e solo com boa umidade. O milho estava com 5 a 6 folhas expandidas, apresentando colar, lígula e aurículas visíveis, com altura do dossel entre de 60 a $70 \mathrm{~cm}$..

Todas as parcelas foram mantidas sem plantas daninhas até o fechamento da cultura, com a eliminação manual de eventuais "escapes" do controle químico e a remoção (capina) de todas as plantas daninhas na testemunha sem herbicida.

As injúrias visuais nas plantas de milho foram avaliadas aos 9 e 16 dias após a aplicação (DAA) dos herbicidas, por meio de escala de notas de 0 a $100 \%$, em que zero representa a ausência de injúrias visuais e 100 a morte da planta (SBCPD, 1995).

No estádio de florescimento e polinização $\left(\mathrm{R}_{1}\right)$, aos 39 DAA, foram determinados os teores foliares de nitrogênio $(\mathrm{N})$, fósforo $(\mathrm{P})$, potássio $(\mathrm{K})$, cálcio $(\mathrm{Ca})$, magnésio $(\mathrm{Mg})$, enxofre $(\mathrm{S})$, boro $(\mathrm{B})$, cobre $(\mathrm{Cu})$, ferro $(\mathrm{Fe})$, manganês $(\mathrm{Mn})$ e zinco $(\mathrm{Zn})$. Foi coletada a primeira folha abaixo da espiga de dez plantas escolhidas aleatoriamente dentro da área útil de cada parcela. O material foi levado para laboratório e analisado por meio de extração via seca, exceto o nitrogênio que foi analisado pelo método de Kjeldahl.

Para a obtenção da produção de grãos de milho, aos 118 DAA, as espigas de duas linhas por seis metros de comprimento foram colhidas manualmente, sendo posteriormente debulhadas, pesadas e a umidade corrigida para $13,5 \%$. Nas linhas colhidas, foi feita a contagem de plantas para determinação da população de milho. O número de espigas, a massa de grãos por espiga e a massa de 400 grãos também foram avaliados.

Os resultados obtidos foram submetidos ao teste $\mathrm{F}$ da análise de variância. Os tratamentos, quando significativo $(\mathrm{p}<0,01$ e $\mathrm{p}<0,05)$, foram comparados pelo teste de Skott-Knott a 5\% de probabilidade. 


\section{Resultados e Discussão}

Os tratamentos estudados não afetaram os teores foliares de N, P, K, Ca, Mg, S, B, Mn e Zn nas plantas de milho (Tabela 1). Porém, houve diferença significativa entre eles para $\mathrm{Fe}$ e $\mathrm{Cu}$. As plantas tratadas com atrazine mais nicosulfuron tiveram maior teor de $\mathrm{Fe}$ nas folhas, e aquelas pulverizadas com glyphosate (nas três dosagens estudadas) e atrazine, maior acúmulo de $\mathrm{Cu}$ nas plantas (Tabela 2). Contudo, mesmo para os tratamentos que resultaram nos menores teores foliares de $\mathrm{Cu}$ e $\mathrm{Fe}$, os valores ficaram dentro da faixa de referência considerados adequados para a cultura o milho (6 - $20 \mathrm{mg} \mathrm{kg}^{-1}$ de $\mathrm{Cu}$ e 20 - $250 \mathrm{de} \mathrm{mg} \mathrm{kg}^{-1}$ de Fe) (MARTINEZ; CARVALHO; SOUZA, 1999).

Tabela 1. Resultados do teste $\mathrm{F}$ da análise de variância para teores de nitrogênio $(\mathrm{N})$, fósforo $(\mathrm{P})$, potássio $(\mathrm{K})$, cálcio $(\mathrm{Ca})$, magnésio $(\mathrm{Mg})$, enxofre $(\mathrm{S})$, boro $(\mathrm{B})$, cobre $(\mathrm{Cu})$, ferro $(\mathrm{Fe})$, manganês $(\mathrm{Mn})$ e zinco $(\mathrm{Zn})$ nas folhas de milho transgênico submetido a diferentes tratamentos de herbicidas. Jaboticabal, SP. 2010/2011.

\begin{tabular}{|c|c|c|c|c|c|c|c|c|c|c|c|}
\hline $\begin{array}{c}\text { Fontes de } \\
\text { variação }\end{array}$ & $\mathbf{N}$ & $\mathbf{P}$ & $\mathbf{K}$ & $\mathrm{Ca}$ & Mg & $\mathbf{S}$ & B & $\mathrm{Cu}$ & $\mathrm{Fe}$ & Mn & $\mathbf{Z n}$ \\
\hline Tratamentos & 1,20 & 0,95 & 1,70 & 0,33 & 0,22 & 0,86 & 1,66 & $2,07 *$ & $2,26^{*}$ & 0,87 & 1,03 \\
\hline Bloco & 0,86 & $22,58 * *$ & 2,32 & $3,11 *$ & 1,86 & 0,67 & 0,58 & 0,34 & 14,35 & 4,13 & $6,44 * *$ \\
\hline $\mathrm{CV}(\%)$ & 5,95 & 5,81 & 5,76 & 14,35 & 12,84 & 11,53 & 15,87 & 9,25 & 12,99 & 24,65 & 7,50 \\
\hline \multirow{2}{*}{ Média geral } & \multicolumn{5}{|c|}{$\left(\mathrm{g} \mathrm{kg}^{-1}\right)$} & & & \multicolumn{4}{|c|}{$\left(\mathrm{mg} \mathrm{kg}^{-1}\right)$} \\
\hline & 28,22 & 2,73 & 27,06 & 2,84 & 0,54 & 1,92 & 6,73 & 10,84 & 201,71 & 26,93 & 15,38 \\
\hline
\end{tabular}

**, * Significativo aos níveis de $1 \%$ e $5 \%$ de probabilidade, respectivamente, pelo teste $\mathrm{F}$ da análise de variância.

Fonte: Elaboração dos autores.

Tabela 2. Teores foliares de ferro $(\mathrm{Fe})$ e cobre $(\mathrm{Cu})$, fitointoxicação aos 9 dias após a aplicação, população de plantas por hectare, massa de 400 grãos e produção de grãos de milho pulverizado com diferentes tratamentos de herbicidas, além da testemunha sem herbicida. Jaboticabal, SP. 2010/2011.

\begin{tabular}{|c|c|c|c|c|c|c|c|}
\hline \multirow{2}{*}{$\begin{array}{l}\text { Herbicidas/ } \\
\text { Testemunha }\end{array}$} & \multirow{2}{*}{$\begin{array}{c}\text { Doses } \\
\left(\mathrm{g} \text { i.a./e.a ha } \text { ha }^{-1}\right)\end{array}$} & $\mathbf{F e}$ & $\mathrm{Cu}$ & \multirow{2}{*}{$\begin{array}{c}\text { Fitointo- } \\
\text { xicação } \\
(\%)\end{array}$} & \multirow{2}{*}{$\begin{array}{l}\text { População } \\
\text { (uni.ha) }\end{array}$} & \multirow{2}{*}{$\begin{array}{c}\text { Massa } 400 \\
\text { grãos } \\
\text { (g) }\end{array}$} & \multirow{2}{*}{$\begin{array}{c}\text { Produção } \\
\text { de grãos } \\
\left(\mathrm{kg} \mathrm{ha}^{-1}\right)\end{array}$} \\
\hline & & \multicolumn{2}{|c|}{$\left(\mathrm{mg} \mathrm{kg}{ }^{-1}\right)$} & & & & \\
\hline Glyphosate & 720 & $184,75 b^{(1)}$ & $12,00 \mathrm{a}$ & $0,00 \mathrm{a}$ & $65219,91 \mathrm{~b}$ & $136,94 \mathrm{a}$ & $9933,44 \mathrm{a}$ \\
\hline Glyphosate & 1200 & $200,00 \mathrm{~b}$ & $11,75 \mathrm{a}$ & $0,00 \mathrm{a}$ & $66435,19 \mathrm{~b}$ & $132,74 \mathrm{~b}$ & $9511,39 \mathrm{~b}$ \\
\hline Glyphosate & $960+720$ & $190,50 \mathrm{~b}$ & $11,75 \mathrm{a}$ & $0,00 \mathrm{a}$ & $67853,01 \mathrm{~b}$ & $133,28 \mathrm{~b}$ & $10742,59 \mathrm{a}$ \\
\hline Atrazine & 2500 & $193,50 \mathrm{~b}$ & $11,62 \mathrm{a}$ & $1,25 \mathrm{a}$ & $71701,39 \mathrm{a}$ & $132,67 \mathrm{~b}$ & $10029,79 \mathrm{a}$ \\
\hline Nicosulfuron & 60 & $194,00 \mathrm{~b}$ & $10,25 \mathrm{~b}$ & $2,50 \mathrm{~b}$ & $72309,03 \mathrm{a}$ & $130,99 \mathrm{~b}$ & $9587,29 \mathrm{~b}$ \\
\hline Mesotrione & 192 & $189,50 \mathrm{~b}$ & $10,00 \mathrm{~b}$ & $0,00 \mathrm{a}$ & $64814,82 \mathrm{~b}$ & $136,58 \mathrm{a}$ & $10824,22 \mathrm{a}$ \\
\hline Tembotrione & 100,8 & $200,25 \mathrm{~b}$ & $10,50 \mathrm{~b}$ & $5,00 \mathrm{~b}$ & $68258,10 \mathrm{~b}$ & $131,37 \mathrm{~b}$ & $9581,66 \mathrm{~b}$ \\
\hline $\begin{array}{l}\text { Atrazine }+ \\
\text { glyphosate }\end{array}$ & $1000+960$ & $208,50 \mathrm{~b}$ & $10,50 \mathrm{~b}$ & $2,50 \mathrm{~b}$ & 69473,38 a & $141,62 \mathrm{a}$ & $10220,44 \mathrm{a}$ \\
\hline $\begin{array}{l}\text { Atrazine+ } \\
\text { nicosulfuron }\end{array}$ & $1000+20$ & $257,50 \mathrm{a}$ & $10,25 \mathrm{~b}$ & $1,25 \mathrm{a}$ & 69878,49 a & $128,41 \mathrm{~b}$ & $9404,97 \mathrm{~b}$ \\
\hline $\begin{array}{l}\text { Atrazine+ } \\
\text { mesotrione }\end{array}$ & $1000+144$ & $186,25 \mathrm{~b}$ & $10,50 \mathrm{~b}$ & $1,25 \mathrm{a}$ & $64814,82 \mathrm{~b}$ & $136,83 \mathrm{a}$ & $10058,80 \mathrm{a}$ \\
\hline $\begin{array}{l}\text { Atrazine }+ \\
\text { tembotrione }\end{array}$ & $1000+75,6$ & $215,00 \mathrm{~b}$ & $10,75 \mathrm{~b}$ & $3,75 \mathrm{~b}$ & 69068,29 a & $131,67 \mathrm{~b}$ & $10264,04 \mathrm{a}$ \\
\hline $\begin{array}{c}\text { Testemunha } \\
\text { sem herbicida }\end{array}$ & & $200,75 \mathrm{~b}$ & $10,25 \mathrm{~b}$ & $0,00 \mathrm{a}$ & $71498,84 \mathrm{a}$ & $138,69 \mathrm{a}$ & $10419,84 \mathrm{a}$ \\
\hline
\end{tabular}

(1) Grupo de médias seguidas pela mesma letra na coluna não diferem entre si a 5\% de probabilidade, pelo teste Scott-Knott.

Fonte: Elaboração dos autores. 
A aplicação de subdoses para simulação de deriva de glyphosate em girassol (Helianthus annuus L.) causou redução nos teores de foliares de $\mathrm{Fe}, \mathrm{Mn}, \mathrm{Zn}$ e $\mathrm{Cu}$ (EKER et al., 2006). O mesmo ocorreu para N, $\mathrm{P}, \mathrm{K}, \mathrm{Cu}$ e $\mathrm{Zn}$ em plantas de café expostas à deriva de glyphosate, aos 45 DAA (FRANÇA et al., 2010). Mas, houve aumento nos teores de K em plantas de pínus (Pinus taeda L.) após a simulação de deriva com glyphosate (SWORD; TIARKS; HAYWOOD, 1998). Plantas de azevém (Lolium multiflorum L.) resistentes ao glyphosate acumularam mais $\mathrm{K}$ e $\mathrm{P}$ que as plantas sensíveis após o tratamento com o herbicida (FERREIRA et al., 2008).

Os tratamentos influenciaram significativamente nas notas de fitointoxicação aos 9 DAA, na população de plantas por hectare, na massa de 400 grãos e na produtividade de grãos (Tabela 3). Por outro lado, não houve diferença entre eles para número de espigas por hectare e massa de grãos por espigas.

Os herbicidas glyphosate e mesotrione isolados não causaram nenhum sintoma visível de fitointoxicação ao milho. Os demais herbicidas ocasionaram injúrias visuais muito leves $(<5 \%)$ às plantas, que não foram mais observadas aos 16 DAA. Os tratamentos que promoveram maior fitointoxicação visual, aos 9 DAA, foram nicosulfuron $(2,5 \%)$ e tembotrione $(5 \%)$ isolados e as misturas atrazine mais glyphosate $(2,5 \%)$ e atrazine mais tembotrione $(3,75 \%)$.

Tabela 3. Resultados do teste $\mathrm{F}$ da análise de variância para notas de fitointoxicação aos 9 dias após a aplicação, população de plantas, número de espigas, massa de grãos por espiga, massa de 400 grãos e produção de grãos de milho submetido a diferentes tratamentos de herbicidas. Jaboticabal, SP. 2010/2011.

\begin{tabular}{|c|c|c|c|c|c|c|c|c|}
\hline \multirow{2}{*}{$\begin{array}{l}\text { Fontes de } \\
\text { variação }\end{array}$} & \multirow{2}{*}{$\begin{array}{l}\text { Fitoin- } \\
\text { toxicação }\end{array}$} & \multirow{2}{*}{$\begin{array}{l}\text { População } \\
\text { de plantas } \\
\text { por hectare }\end{array}$} & \multicolumn{2}{|c|}{ Número de espigas } & \multirow{2}{*}{$\begin{array}{l}\text { Número de } \\
\text { grãos por } \\
\text { espiga }\end{array}$} & \multirow{2}{*}{$\begin{array}{c}\text { Massa } \\
\text { de } \\
\text { grãos } \\
\text { por } \\
\text { espiga }\end{array}$} & \multirow{2}{*}{$\begin{array}{c}\text { Massa } \\
\text { de } \\
400 \\
\text { grãos }\end{array}$} & \multirow{2}{*}{$\begin{array}{l}\text { Produção } \\
\text { de grãos por } \\
\text { hectare }\end{array}$} \\
\hline & & & $\begin{array}{c}\text { Por } \\
\text { planta }\end{array}$ & $\begin{array}{c}\begin{array}{c}\text { Por } \\
\text { hectare }\end{array} \\
\end{array}$ & & & & \\
\hline Tratamentos & $3,61 * *$ & $2,38^{*}$ & 1,08 & 0,76 & 1,12 & 1,38 & $2,04 *$ & $2,32 *$ \\
\hline Bloco & 2,47 & 2,70 & 0,41 & 2,34 & 0,39 & 1,16 & $10,11 * *$ & $13,66^{* *}$ \\
\hline CV (\%) & 20,60 & 5,09 & 7,25 & 8,59 & 7,92 & 8,94 & 3,96 & 6,33 \\
\hline \multirow{2}{*}{ Média geral } & $(\%)$ & $\left(\right.$ uni.ha $\left.^{-1}\right)$ & (uni.) & (uni.) & (uni.) & (g) & (g) & $\left(\mathrm{kg} \mathrm{ha}^{-1}\right)$ \\
\hline & 1,46 & 68443,77 & 0,76 & 59239,97 & 506,05 & 169,91 & 134,32 & 10025,70 \\
\hline
\end{tabular}

**, * Significativo aos níveis de $1 \%$ e $5 \%$ de probabilidade, respectivamente, pelo teste $\mathrm{F}$ da análise de variância.

Fonte: Elaboração dos autores.

Nas parcelas da testemunha sem aplicação e naquelas pulverizadas com atrazine e nicosulfuron isolados e com as misturas de atrazine com glyphosate, nicosulfuron ou tembotrione houve maior população de plantas de milho. Para alguns tratamentos, como mesotrione isolado e em mistura com atrazine, a menor densidade de plantas não refletiu na produção de grãos, pois houve uma compensação pela maior massa de grãos. A esse respeito, as plantas de milho da testemunha $\mathrm{e}$ as plantas tratadas com glyphosate a $720 \mathrm{~g} \mathrm{ha}^{-1}$ e mesotrione isolados e com as associações de atrazine ao glyphosate ou mesotrione tiveram maior massa de 400 grãos.

Para a testemunha sem herbicida, os tratamentos isolados de atrazine, mesotrione e glyphosate (a $720 \mathrm{~g} \mathrm{ha}^{-1}$ e sequencial) e as misturas de atrazine com glyphosate, mesotrione ou tembotrione houve maior produção de grãos de milho. As plantas pulverizadas com glyphosate a $1200 \mathrm{~g}^{-}$ ${ }^{1}$, nicosulfuron a $60 \mathrm{~g} \mathrm{ha}^{-1}$, tembotrione a $100,8 \mathrm{~g}$ $\mathrm{ha}^{-1} \mathrm{e}$ atrazine mais nicosulfuron (1000 mais $20 \mathrm{~g}$ 
ha $^{-1}$ ) tiveram redução média de $8,8 \%$ na produção. Embora a fitointoxicação visual ocasionada por esses herbicidas tenha sido nula (glyphosate) ou muito baixa (nicosulfuron, tembotrione e atrazine + nicosulfuron) houve efeito negativo na produção de grãos. Possivelmente, a resposta fisiológica das plantas de milho a esses herbicidas esteja relacionada a outros fatores não estudados nesse trabalho.

Não foram encontradas literaturas que justificassem o efeito diferencial do glyphosate, em aplicação única e sequencial ou em mistura com atrazine, na produção do milho tolerante a esse herbicida. O glyphosate em mistura com atrazine $\left(960+1000 \mathrm{~g} \mathrm{ha}^{-1}\right)$, em aplicação única a $720 \mathrm{~g} \mathrm{ha}^{-1}$ ou sequencial (960 mais $720 \mathrm{~g} \mathrm{ha}^{-1}$ ) não prejudicou a produtividade do milho, mas, com a dose única de $1200 \mathrm{~g} \mathrm{ha}^{-1}$ houve redução. As respostas fisiológicas e produtivas das plantas de milho transgênico tratadas com glyphosate devem ser melhor estudadas, para entendimento dos processos inibidos.

O efeito fitotóxico de glyphosate em soja transgênica ainda é bastante discutido e os resultados bem variáveis. Apesar de redução no conteúdo de clorofila, biomassa de nódulos e conteúdo de legmoglobina (REDDY; HOAGLAND; ZABLOTOWICZ, 2000) e fixação e acúmulo de nitrogênio nas raízes (KING; PURCELL; VORIES, 2001) de algumas variedades transgênicas, a maioria dos trabalhos comprovaram ausência de efeito significativo na produtividade de grãos (CORREIA; DURIGAN, 2007; CORREIA; DURIGAN, 2010; NORSWORTHY, 2004; NURSE et al., 2007; ZABLOTOWICZ; REDDY, 2007).

Outros trabalhos comprovaram o efeito tóxico dos herbicidas nicosulfuron e tembotrine em plantas de milho. Entre seis híbridos de milho doce avaliados, o Merit foi o único com extrema sensibilidade ao tembotrione, que ocasionou a mortalidade das plantas e, consequentemente, perda total da produção (BOLLMAN et al., 2008). O híbrido de milho B761 teve $17,4 \%$ de redução na produção de grãos devido à exposição ao nicosulfuron (60 $\left.\mathrm{g} \mathrm{ha}^{-1}\right)$, entre cinco genótipos testados (CAVALIERI et al., 2008). Em outro estudo, a produtividade do milho foi dependente da dose aplicada de nicosulfuron (60 ou $80 \mathrm{~g} \mathrm{ha}^{-1}$ ) nos estádios de desenvolvimento $\mathrm{V}_{6}$ e $\mathrm{V}_{9}$, mas, não foi afetada pelo herbicida quando este foi pulverizado em plantas no estádio $\mathrm{V}_{3}$ (SPADER; VIDAL, 2001). Os autores concluíram que a seletividade do nicosulfuron para a cultura do milho diminui a partir do estádio $\mathrm{V}_{6}$ e com o aumento da dose acima de $60 \mathrm{~g} \mathrm{ha}^{-1}$. Portanto, o estádio de desenvolvimento do milho no momento da aplicação e o material genético utilizado influenciam diretamente na ação dos herbicidas nas plantas.

Com base nos resultados obtidos, concluiu-se que os tratamentos de herbicidas estudados não afetaram negativamente os teores foliares de $\mathrm{N}$, $\mathrm{P}, \mathrm{K}, \mathrm{Ca}, \mathrm{Mg}, \mathrm{S}, \mathrm{B}, \mathrm{Mn}$ e Zn nas plantas de milho (híbrido DKB 390 RR). Além disso, as aplicações isoladas de glyphosate (1200 $\left.\mathrm{g} \mathrm{ha}^{-1}\right)$, nicosulfuron, tembotrione e da mistura atrazine mais nicosulfuron ocasionaram redução na produção de grãos de milho.

\section{Referências}

AGROFIT - SISTEMA DE AGROTÓXICOS FITOSSANITÁRIOS. Consulta a produtos formulados. 2011. Disponível em: <http://extranet.agricultura.gov. br/agrofit_cons/principal_agrofit_cons $>$. Acesso em: 15 nov. 2011 .

BELLALOUI, N.; ROBERT, M.; ZABLOTOWICZ, R. M.; REDDY, K. N.; ABEL, C. A. Nitrogen metabolism and seed composition as influenced by glyphosate application in glyphosate-resistant soybean. Journal of Agricultural and Food Chemistry, Washington, v. 56, n. 8, p. 2765-2772, 2008.

BOLLMAN, J. D.; BOERBOOM, C. M.; BECKER, R. L.; FRITZ, V. A. Efficacy and tolerance to HPPDinhibiting herbicides in sweet corn. Weed Technology, Champaign, v. 22, n. 4, p. 666-674, 2008.

CAKMAK, I.; YAZICI, A.; TUTUS, Y.; OZTURK, L. Glyphosate reduced seed and leaf concentrations of calcium, manganese, magnesium, and iron in nonglyphosate resistant soybean. European Journal of Agronomy, Montpellier, v. 31, n. 3, p. 114-119, 2009. 
CAVALIERI， S. D.; OLIVEIRA JUNIOR, R. S.; CONSTANTIN, J.; BIFFE, D. F.; RIOS, F. A.; FRANCHINI, L. H. M. Tolerância de híbridos de milho ao herbicida nicosulfuron. Planta Daninha, Viçosa, MG, v. 26, n. 1, p. 203-214, 2008.

CORREIA, N. M.; DURIGAN, J. C. Seletividade de diferentes herbicidas à base de glyphosate a soja RR. Planta Daninha, Viçosa, MG, v. 25, n. 2, p. 375-379, 2007.

Controle de plantas daninhas na cultura da soja resistente ao glyphosate. Bragantia, Campinas, n. 69, n. 2, p. 319-327, 2010.

EKER, S.; OZTURK, L.; YAZICI, A.; ERENOGLU, B.; ROMHELD, V.; CAKMAK, I. Foliar-applied glyphosate substantially reduced uptake and transport of iron and manganese in sunflower (Helianthus annuus L.) plants. Journal of Agricultural and Food Chemistry, Washington, v. 54, n. 26, p. 10019-10025, 2006.

FERREIRA, E. A.; SILVA, A. A.; REIS, M. R.; SANTOS, J. B.; OLIVEIRA, J. A.; VARGAS, L.; KHOURI, K. R.; GUIMARÃES, A. A. Distribuição de glyphosate e acúmulo de nutrientes em biótipos de azevém. Planta Daninha, Viçosa, MG, v. 26, n. 1, p. 165-173, 2008.

FRANÇA, A. C.; FREITAS, M. A. M.; D’ANTONINO, L; FIALHO, C. M. T.; SILVA, A. A.; REIS, M. R.; RONCHI, C. P. Teores de nutrientes em cultivares de café arábica submetidos à deriva de glyphosate. Planta Daninha, Viçosa, MG, v. 28, n. 4, p. 877-885, 2010.

KING, C. A.; PURCELL, L. C.; VORIES, E. D. Plant growth and nitrogenase activity of glyphosate-tolerant soybean in response to foliar glyphosate applications. Agronomy Journal, Madison, v. 93, n. 1, p. 179-186, 2001.

MARTINEZ, H. E. P.; CARVALHO, J. G.; SOUZA, R. B. Diagnose foliar. In: RIBEIRO, A. C.; GUIMARÃES, P. T. G.; ALVAREZ, V. V. H. (Ed.). Recomendações para uso de corretivos e fertilizantes em Minas Gerais: $5^{\text {a }}$ aproximação. Viçosa: Comissão de Fertilidade do Solo do Estado de Minas Gerais, 1999. p. 143-168

NORSWORTHY, J. K. Broadleaved weed control in wide-row soybean (Glycine max) using conventional and glyphosate herbicide programmes. Crop Protection, Guildford, v. 23, n. 12, p. 1229-1235, 2004.
NURSE, R. E.; HAMILL, A. S.; SWANTON, C. J.; SWANTON, C. J.; TARDIF, F. J.; DEEN, W.; SIKKEMA, P. H. Is the application of a residual herbicide required prior to glyphosate application in no-till glyphosatetolerant soybean (Glycine max)? Crop Protection, Guildford, v. 26, v. 4, p. 484-489, 2007.

REDDY, K. N.; HOAGLAND, R. E.; ZABLOTOWICZ, R. M. Effect of glyphosate on growth, chlorophyll, and nodulation in glyphosate-resistant and susceptible soybean (Glycine max) varieties. Journal of New Seeds, Binghamton, v. 2, n. 1, p. 37-52, 2000.

REDDY, K.; BELLALOUI, N.; ZABLOTOWICZ, R. Glyphosate Effect on shikimate, nitrate reductase activity, yield, and seed composition in corn. Journal of Agricultural and Food Chemistry, Washington, v. 58, n. 6, p. 3646-3650, 2010.

SANTOS, J. B.; FERREIRA, E. A.; REIS, M. R.; SILVA, A. A.; FIALHO, C. M. T.; FREITAS, M. A. M. Avaliação de formulações de glyphosate sobre soja Roundup Ready. Planta Daninha, Viçosa, MG, v. 25, n. 1, p. 165171, 2007.

SERRA, A. P.; MARCHETTI, M. E.; CANDIDO, A. C. S.; DIAS, A. C. S.; CHRISTOFFOLETI, P. J. Influência do glifosato na eficiência nutricional do nitrogênio, manganês, ferro, cobre e zinco em soja resistente ao glifosato. Ciência Rural, Santa Maria, v. 41, n. 1, p. 7784, 2011.

SOCIEDADE BRASILEIRA DA CIÊNCIA DAS PLANTAS DANINHAS - SBCPD. Procedimentos para instalação, avaliação e análise de experimentos com herbicidas. Londrina: SBCPD, 1995. 42 p.

SPADER, V.; VIDAL, R. A. Seletividade e dose de injúria econômica de nicosulfuron aplicado em diferentes estádios de desenvolvimento da cultura do milho. Ciência Rural, Santa Maria, v. 31, n. 6, p. 929-934, 2001.

SWORD, M. A.; TIARKS, A. E.; HAYWOOD, J. D. Establishment treatments affect relationships among nutrition, productivity and competing vegetation of loblolly pine saplings on a Gulf Coastal Plain site. Forest Ecology and Management, Amsterdam, v. 105, n. 2, p. 175-188, 1998.

ZABLOTOWICZ, R. M.; REDDY, K. N. Nitrogenase activity, nitrogen content, and yield responses to glyphosate in glyphosate-resistant soybean. Crop Protection, Guildford, v. 26, n. 3, p. 370-376, 2007. 
